\title{
In silico and in vitro analysis of rare germline allelic variants of RET oncogene associated with medullary thyroid cancer
}

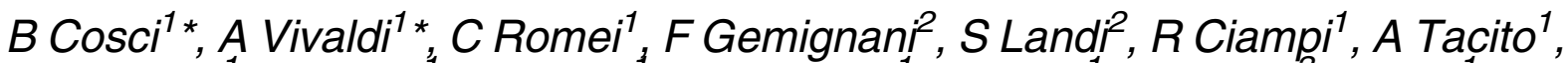

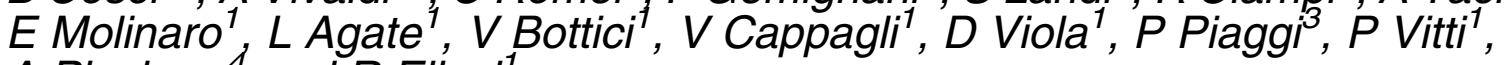 \\ A Pinchera ${ }^{4}$ and $R$ Elisei ${ }^{1}$
}

\author{
${ }^{1}$ Department of Endocrinology and Metabolism, ${ }^{2}$ Department of Biology, ${ }^{3}$ Department of Energy and Systems Engineering and \\ ${ }^{4}$ AMBISEN Center, High Technology Center for the Study of the Environmental Damage of the Endocrine and Nervous Systems, \\ University of Pisa, Via Paradisa 2, 56124 Pisa, Italy \\ (Correspondence should be addressed to R Elisei; Email: relisei@endoc.med.unipi.it) \\ *(B Cosci and A Vivaldi contributed equally to this work)
}

\begin{abstract}
Germline and somatic RET oncogene mutations are found in $98 \%$ hereditary and $40 \%$ sporadic medullary thyroid carcinomas. Our aim was to analyse by in silico and in vitro assays the transforming activity of six rare RET mutations (T338I, V648I, M918V, A883T, S904F and M848T). Six known RET mutations were used as controls. The in silico analysis showed the highest score value (i.e. 65) for S904F, M848T, M918T and C634R, whereas L790F, G691S, T338I and V648I had 0 score. Intermediate score values were obtained by A883T (score $=55$ ), M918V, V804M and Y791F (score=15). The in vitro focus formation assay showed that cells transfected with S904F, M918T, M848T or C634R generated the largest number of focus formation units (FFU). Intermediate numbers of FFU were observed in cells transfected with M918V, V804M, Y791F or A883T, while cells transfected with L790F, G691S, T338I or V648I showed a number of FFU similar to control cells. A positive correlation between the in silico score and in vitro FFU was found $(P=0.0005)$. Only cells transfected with M918T or C634R grew faster and generated higher number of colonies in soft agar than control cells. However, the cells that were transfected with V804M produced an intermediate number of colonies. In conclusion, two of the six rare RET mutations, S904F and M848T possessed a relatively high transforming activity but a low aggressiveness; the other four mutations T338I, V648I, M918V and A883T were low or non-transforming, and their ability to induce tumoural transformation might be related to particular genetic conditions.
\end{abstract}

Endocrine-Related Cancer (2011) 18 603-612

\section{Introduction}

The human RET gene maps to chromosome 10q11.2 and is composed of 21 exons with a size of about $55 \mathrm{~kb}$ (Pasini et al. 1995). The gene encodes a tyrosine kinase transmembrane receptor (Takahashi et al. 1988, Machens et al. 2009) characterised by three different domains: the extracellular domain, the transmembrane domain and the intracellular tyrosine kinase domain.

In 1993, independent groups discovered that germline point mutations in the proto-oncogene are the causative events in hereditary and sporadic medullary thyroid cancer (MTC) (Donis-Keller et al. 1993, Mulligan et al. 1993, Eng et al. 1994). After the introduction of genetic screening in the diagnostic procedures of patients affected with apparently sporadic MTC, new mutations were found, especially in noncysteine-rich regions (Eng et al. 1995a, Erdogan et al. 2005, Elisei et al. 2007), that were mainly associated with the isolated form of familial MTC (FMTC) (Romei et al. 2011). In fact, from 1993 to 2008, a total of 179 point mutations and 66 deletion/insertion mutations have been described (www.hgmd.cf.ac.uk).

During 18 years of genetic screening (1993-2011), we found six germline mutations associated with apparently sporadic MTC, which were unreported at the time of our discovery. Although nowadays 
all of them have been described as associated with MTC (Nunes et al. 2002, Romei et al. 2011), only the functional activity of the A883T mutation has been published (Elisei et al. 2004). Thus, since the functional activity of five out of these six rare RET mutations has never been studied they will be indicated as 'new' RET mutations in contrast with the old well known other RET mutations used as controls.

Although associated with a clinical phenotype, such as the MTC, all new proto-oncogene mutations must be analysed by in vitro studies to define their transforming ability (Mise et al. 2006). Recently, however, a new computerised method based on the alignment of both the wild type and mutant sequences of the oncogene (i.e. in silico analysis) has been developed, and it is proposed as a valid alternative to the in vitro analysis for the determination of the transforming ability of the mutated gene (Mathe et al. 2006, Tavtigian et al. 2008).

The aim of this study was to analyse the transforming ability of these six rare RET mutations, by both in vitro and in silico methods and to correlate the results with the clinical aggressiveness of the MTC associated to each one of them.

\section{Materials and methods}

\section{Patients}

A total of 1389 subjects were subjected to genetic screening for the last 18 years at the Department of Endocrinology of Pisa University (Italy). Epidemiological features and reasons for genetic screening are reported in Table 1. An informed consent to genetic screening and other clinical procedures was signed by both MTC and control patients. This study was approved by the Institutional Review Board. Of note, the 200 healthy volunteers that were selected as control patients were not relatives of the MTC patients. Control cases were matched with MTC patients by age, sex and geographic area of birth. The diagnosis of MTC was based on the histopathology of the thyroid tumours after thyroidectomy.

\section{Genomic DNA extraction, PCR amplification and sequencing}

Blood was collected in EDTA from all subjects and stored at $-20^{\circ} \mathrm{C}$. Initially, genomic DNA was extracted using a homemade phenol-chloroform method, but in later years a more automated method was used, as described previously (Elisei et al. 2007). Recently, DNA was purified from $400 \mu$ whole blood using an easy and efficient automated method
Table 1 Clinical features of subjects subjected to RET genetic screening between 1993 and 2011 at the Department of Endocrinology, University of Pisa, Italy

\begin{tabular}{lccl}
\hline $\begin{array}{l}\text { Reason for } \boldsymbol{R E T} \\
\text { genetic screening }\end{array}$ & $\begin{array}{c}\text { Number of } \\
\text { subjects }\end{array}$ & Sex M/F & $\begin{array}{l}\text { Average } \\
\text { age M/F } \\
\text { (years) }\end{array}$ \\
\hline Apparently, sporadic MTC & $764^{\mathrm{a}}$ & $323 / 441$ & $52 / 50$ \\
Index case of hereditary MTC & 56 & $22 / 34$ & $40 / 36$ \\
Relatives of familial MTC & 369 & $169 / 200$ & $39 / 37$ \\
Normal subjects ${ }^{\text {b }}$ & 200 & $66 / 134$ & $50 / 49$ \\
Total & 1389 & $580 / 809$ & $45 / 43$ \\
\hline
\end{tabular}

${ }^{\text {a}}$ Forty-seven subjects were found to carry an unexpected $R E T$ germline mutation.

${ }^{5}$ Normal subjects were screened as controls, with their informed consent.

(Maxwell 16 Instrument, Promega), eluted in $300 \mu \mathrm{l}$ water and stored at $-20^{\circ} \mathrm{C}$. For all screened subjects an aliquot of DNA was stored separately at $-20{ }^{\circ} \mathrm{C}$ with the intent of analysing for the presence of new mutations whenever described.

The gene exons $5,8,10,11,13,14,15$ and 16 were analysed in all subjects using a touchdown (TD) PCR assay (Hecker \& Roux 1996) and the HotStarTaq PCR kit (Qiagen, Courtaboeuf, France) in a final volume of $50 \mu \mathrm{l}$ (My Cycle Instrument, Bio-Rad). TD amplification was performed with an initial denaturation step at $95{ }^{\circ} \mathrm{C}$ for $15 \mathrm{~min}$, followed by three cycles of denaturation at $95^{\circ} \mathrm{C}$ for $30 \mathrm{~s}$, annealing starting at $66^{\circ} \mathrm{C}$ (decreasing by $2{ }^{\circ} \mathrm{C} /$ cycle) for $45 \mathrm{~s}$ and extension at $72{ }^{\circ} \mathrm{C}$ for $1 \mathrm{~min}$. After this step was 34 additional cycles of denaturation at $95^{\circ} \mathrm{C}$ for $30 \mathrm{~s}$, annealing at $55^{\circ} \mathrm{C}$ for $45 \mathrm{~s}$ and extension at $72{ }^{\circ} \mathrm{C}$ for $1 \mathrm{~min}$, followed by a final extension at $72{ }^{\circ} \mathrm{C}$ for $10 \mathrm{~min}$. The amplified DNA was analysed on $2 \%$ agarose gels and purified using a commercial kit (Jetquick purification kit, Euroclone, Milan, Italy). Sequence analysis was performed with an automated system employing fluorescent dye terminators (ABI Prism 3110, PerkinElmer, Foster City, CA, USA). If the amplification of an exon failed using the 'TD' method, then another PCR protocol was implemented using optimised PCR conditions for the exon of interest. Whenever the identified point mutation altered (creating or deleting) the recognition site of a restriction enzyme, we performed restriction analysis of the mutated exon to confirm the presence of the mutation.

\section{In silico analysis}

The in silico analysis was performed by the "AlignGrantham Variation-Grantham Deviation' (AlignGVGD) web-based program (http://agvgd.iarc.fr), which 
combines the biophysical characteristics of amino acids and proteins to predict the degree of alteration of the biological activity of the encoded protein, determined by a missense point mutation. The Align-GVGD method takes into account the composition $(\mathrm{C})$, polarity $(\mathrm{P})$ and volume (V) of amino acids (Tavtigian et al. 2008). Based on multiple sequence alignments (MSA), the programme calculates two scores for each amino acid residue substitution, GD and GV. GV is the measure of the amount of observed biochemical variation in a particular position in the alignment. GD is the measure of the biochemical difference between the mutant and the observed variation at that position according to the MSA. GV and GD values are calculated for eight different MSA: humans, chickens, frogs, rats, mice, cattle, pigs and fish.

Accordingly, the GV and GD value the system predicts the degree of structural difference between the mutated and the wild-type protein. The degree of this difference is expressed as a numerical score ranging from 0 (no difference) to 65 (maximum difference). The assumption is that the higher the score the more powerful the transforming activity.

To verify the bona fide of this electronic calculation we also performed a functional prediction of both new and known mutations by a manual calculation according to the different combination of the $\mathrm{GV}$ and GD values (Mathe et al. 2006).

\section{In vitro analysis}

\section{Site-directed mutagenesis}

Six known (M918T, C634R, V804M, Y791F, L790F and G691S) and six new (M848T, S904F, A883T, M918V, V648I and T338I) missense mutations were introduced into the sequence of the short $3^{\prime}$ splice isoform in RET nine of the human cDNA cloned in the plasmid pRC/CMV (kindly gifted by Prof. Isabella Ceccherini, from Molecular Genetics Laboratory, Institute Giannina Gaslini, Genova, Italy) using the QuikChange XL site-directed mutagenesis kit (Stratagene, La Jolla, CA, USA). The mutagenesis products were submitted to sequence analysis to verify the presence of the RET mutation.

\section{Cell lines transfection and focus formation assay}

NIH 3 T3 mouse fibroblasts were maintained in DMEM supplemented with $10 \%$ foetal bovine serum and $1 \%$ penicillin/streptomycin solution (Sigma). For transfection, cells were plated at the density of $1 \times 10^{5}$ in six-well plates and stably transfected with one of the 12 pRC/CMV plasmids containing a mutagenised gene. As controls, the wild-type gene and empty pRC/CMV vector were transfected in the same cell system.
Transfection was performed with a lipid mixture, Lipofectamine (Invitrogen). Forty-eight hours after transfection, $80 \%$ of cells were distributed among three plates that were cultured without selection and after 2 weeks the transformed foci were counted. A fourth plate was obtained with the remaining $20 \%$ of transfected cells and subjected to selection with $400 \mu \mathrm{g} / \mathrm{ml}$ neomycin for 15 days, and the stably transfected mass cultures were subsequently used for further experiments, such as growth curves and growth in soft agar. The transforming activity of the six new and the six known missense mutations was analysed by counting the number of focusforming units per microgram of DNA (FFU/ $\mu \mathrm{g}$ DNA) transfected in NIH $3 \mathrm{~T} 3$ fibroblasts and comparing it with the number of FFU/ $\mu \mathrm{g}$ DNA spontaneously developed in mock- and wild-type transfected and non-transfected NIH 3T3 control cell lines.

\section{Growth curves}

Each of the 12 NIH 3T3 cell lines stably transfected with the $\mathrm{pRC} / \mathrm{CMV}$ plasmids containing a mutagenised $R E T$ gene, the two transfected controls and the nontransfected NIH 3T3 fibroblasts were plated at $20 \times 10^{4}$ cells per $3.5 \mathrm{~cm}$ dish in triplicate. The cell number was counted after 2 and 4 days of growth; the ratio between the numbers of the cells of each transfected cell line with that of the control cell lines represented the proliferation rate. As an expression of the growth rate, the cell growth doubling time $\left(T_{\mathrm{d}}\right)$ was calculated according to the following formula: $T_{\mathrm{d}}=\left(t_{2}-t_{1}\right) \times \log 2 /(\log \mathrm{N} 2-\log \mathrm{N} 1)$, where $t_{2}$ and $t_{1}$ are different times chosen during the logarithmic phase of cell growth and N2 and N1 are the numbers of cells at those times.

\section{Soft agar assay}

The colony formation ability of the NIH 3T3 stably transfected cells in soft agar was tested by resuspending $2 \times 10^{4}$ cells of each cell line in $1.5 \mathrm{ml}$ culture medium containing $0.4 \%$ low-melting-temperature agar and plating them in triplicate in a six-well plate over a base layer of $1.5 \mathrm{ml}$ culture medium containing $0.75 \%$ lowmelting-temperature agar. The plates were incubated for 4 weeks until colonies were formed. Colonies were stained with $0.005 \%$ crystal violet and counted. The same experiment was performed by the two transfected and the non-transfected NIH 3T3 control cell lines.

\section{Results}

\section{New gene mutations}

After 18 years of screening patients with apparently sporadic MTC, we found six gene point mutations that 
resulted in an amino acid change, which were unreported at the time of our discovery (Table 2). They were all germline mutations, but with a low prevalence because each one of them was found only in one kindred among the 103 families that were studied at our Department (Romei et al. 2011). Moreover, as shown in Table 2, two of the six new mutations, A883T and S904F, were associated with MTC in at least one more family member (i.e. brother and son respectively). Conversely, the other four mutations were associated with MTC only in the index cases, even if they were also identified in other family members who did not yet develop the MTC at the time of this study (Table 2).

In addition, the negative search for these new mutations among 200 normal subjects excluded the possibility that they could be polymorphic variants of the gene.

\section{In silico analysis}

The results of the in silico analysis using the AlignGVGD method are reported in Fig. 1. The T338I and V648I scores were 0; the M918V score was 15; the A883T score was 55 and the S904F and M848T scores were 65 . As a control, the calculated score of some known, highly transforming mutations (i.e. C634R and M918T) was 65. In contrast, the calculated score of other known less transforming mutations was 15 (i.e. V804M and Y791F) and 0 (i.e. L790F). As an additional control, the calculated Align-GVGD score of the G691S missense polymorphism was 0 .

When we reanalysed the functional prediction of our mutations by the manual calculation according with Mathe et al. (2006), we found comparable results but, in our opinion, less defined since all mutations with a score $>0$ were considered to have the same functional activity (Deleterious 1) while those with 0 score showed three different degrees of functional activity (from Neutral 1 to Deleterious 2) (Table 3).

\section{In vitro analysis}

\section{Focus assay}

In Fig. 2, the two well known, highly transforming mutations C634R and M918T showed $>100 \mathrm{FFU} / \mu \mathrm{g}$ DNA. Similarly, the two new mutations, M848T and S904F, had > $100 \mathrm{FFU} / \mu \mathrm{g}$ DNA, suggesting a high transforming activity for both of the new mutations that is comparable to that of C634R and M918T. Intermediate amounts of FFU were observed for the known mutations V804M and Y791F (between 15 and $30 \mathrm{FFU} / \mu \mathrm{g}$ DNA, respectively), for which the transforming activity is usually considered lower than that of C634R and M918T. Similarly, the new mutations A883T and M918V showed between 15 and $30 \mathrm{FFU} / \mu \mathrm{g}$ DNA. Finally, the known mutation (L790F) and the two new mutations (V648I and T338I) had <12.5 FFU/ $\mu$ g DNA, which is similar to that shown by the wild-type RET transfected and the non-transfected NIH 3T3 control cell lines (0.5-12.55 FFU/ $\mu \mathrm{g}$ DNA); therefore, this result suggests a non-transforming activity of these mutations. The same negative result was obtained, as expected, in cells transfected with the G691S missense polymorphism.

\section{Growth curves}

To compare the mitogenic abilities of the NIH $3 \mathrm{~T} 3$ fibroblasts stably transfected with the 12 mutations to that of the NIH 3 T3 cell lines used as controls, growth curves of each cell line were calculated. As shown in Fig. 3, the proliferation rates of cells transfected with C634R, M918T, Y791F, A883T, V648I and T338I were significantly higher $(P<0.05)$ than the proliferation rates of control cell lines. At variance, the

Table 2 New RET mutations found during the RET genetic screening performed at the Department of Endocrinology, University of Pisa (Italy) between 1993 and 2011

\begin{tabular}{|c|c|c|c|c|c|c|}
\hline Codon & Exon & $\begin{array}{c}\text { Wild-type } \\
\text { AA/nucleotides }\end{array}$ & $\begin{array}{c}\text { Mutation } \\
\text { AA/nucleotides }\end{array}$ & Polymorphism & $\begin{array}{c}\text { Familial } \\
\text { members with } \\
\text { mutation }(n)\end{array}$ & $\begin{array}{l}\text { MTC affected } \\
\text { subjects }(n)\end{array}$ \\
\hline 338 & 5 & Thr/ACC & Ile/ATC & No & 2 & 1 \\
\hline 648 & 11 & Val/GTC & Ile/ATC & No & 2 & $1^{a}$ \\
\hline 848 & 14 & Met/ATG & Thr/ACG & No & 2 & 1 \\
\hline 904 & 15 & Ser/TCC & Phe/TTC & No & 2 & 2 \\
\hline 883 & 15 & $\mathrm{Ala} / \mathrm{GCT}$ & $\mathrm{Thr} / \mathrm{ACT}$ & No & 6 & $2^{b}$ \\
\hline 918 new & 16 & Met/ATG & Val/GTG & No & 2 & 1 \\
\hline
\end{tabular}

AA, amino acid; MTC, medullary thyroid cancer; $n$, number of cases.

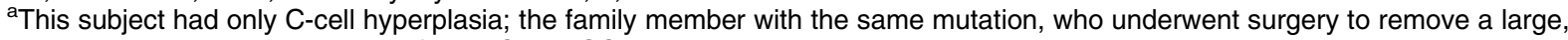
multinodular goitre, was negative for MTC and $\mathrm{CCH}$ upon histological evaluation.

${ }^{\mathrm{b}} \mathrm{MTC}$ was found in only two homozygous cases; the four family members with the same heterozygous mutation did not yet develop the MTC after 7 years of follow-up and an average age of 50 years. 


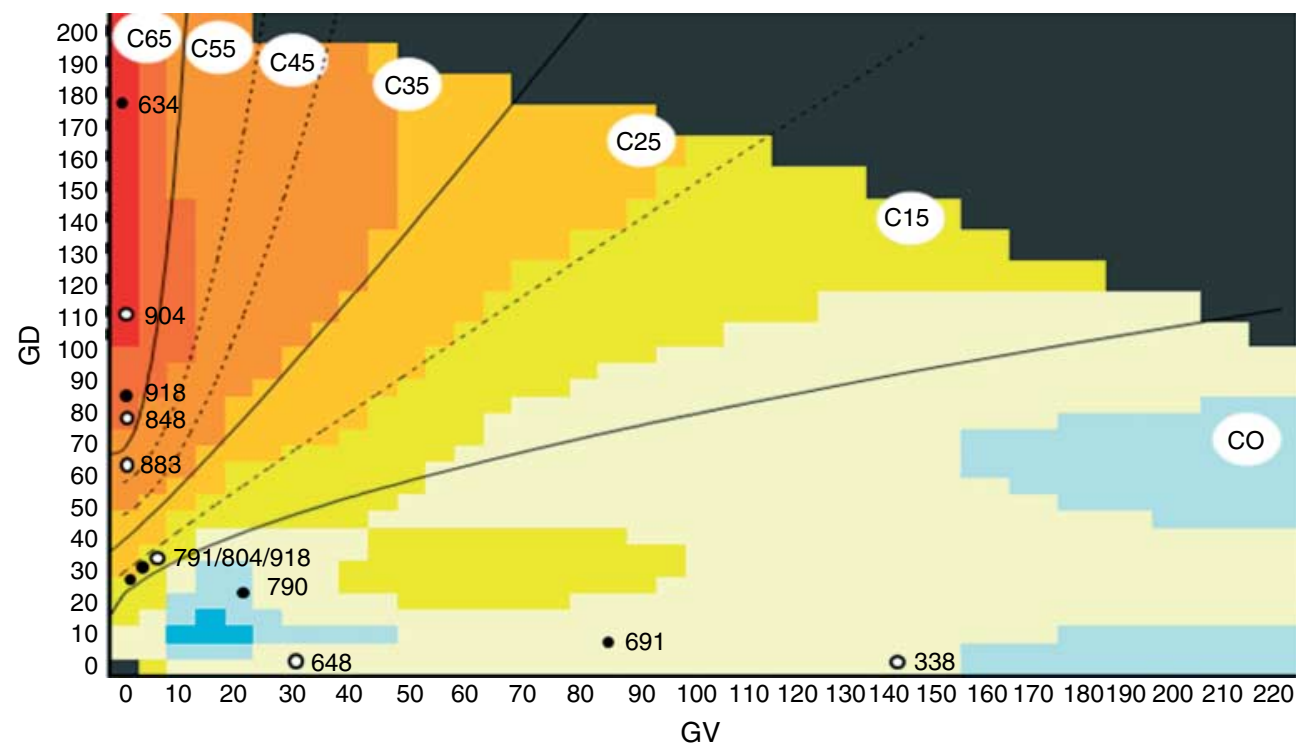

Figure 1 Results of the in silico analysis using the Align-GVGD method. New germline RET mutations are indicated with an open circle, and known RET germline mutations are indicated with a black filled dot. GV, Grantham Variation; GD, Grantham Deviation.

proliferation rates of cells transfected with G691S, L790F, S904F, M918V, M848T and V804M did not show any statistically significant difference with respect to the proliferation rates of control cells. Notably, the proliferation rates of cells transfected with C634R and M918T were significantly higher than the proliferation rates of cells transfected with M848T and S904F, which previously exhibited similar AlignGVGD scores and FFUs.

\section{Soft agar assay}

We assessed the anchorage-independent growth in soft agar of the NIH 3T3 cell lines used as controls and of all the other cell lines transfected with the 12 mutations (Fig. 4). The C634R and M918T mutations produced the largest number of colonies (i.e. 24 and 20 colonies per plate respectively) (Fig. 4 Panel A), and the other known and new mutations produced relatively low number of colonies, which were not much higher than

Table 3 In silico, in vitro and in vivo features of a series of known $(\mathrm{K})$ and new $(\mathrm{N})$ germline RET mutations

\begin{tabular}{|c|c|c|c|c|c|c|c|}
\hline $\begin{array}{l}\text { Mutated } \\
\text { codon }\end{array}$ & $\begin{array}{c}\text { K or } \mathbf{N} \\
\text { mutation }\end{array}$ & $\begin{array}{l}\text { Align GD/GV } \\
\text { score }\end{array}$ & $\begin{array}{c}\text { Align GD/GV } \\
\text { prediction }\end{array}$ & $\begin{array}{c}\text { DNA } \\
(\mathrm{FFU} / \mu \mathrm{g})\end{array}$ & $\begin{array}{c}\text { No. of soft } \\
\text { agar colonies }\end{array}$ & $\begin{array}{l}\text { Doubling } \\
\text { time (days) }\end{array}$ & $\begin{array}{c}\text { ATA } \\
\text { risk levels }\end{array}$ \\
\hline C634R & $\mathrm{K}$ & 65 & Deleterious 1 & $183 \pm 19.8$ & $24 \pm 5$ & $0.6 \pm 0.8$ & C \\
\hline M918T & $\mathrm{K}$ & 65 & Deleterious 1 & $98.5 \pm 9.2$ & $20 \pm 5$ & $0.6 \pm 0.1$ & $\mathrm{D}$ \\
\hline M848T & $\mathrm{N}$ & 65 & Deleterious 1 & $171 \pm 49.5$ & $6 \pm 2$ & $1.5 \pm 0.8$ & $A^{c}$ \\
\hline S904F & $\mathrm{N}$ & 65 & Deleterious 1 & $98 \pm 26.9$ & $9 \pm 5$ & $1.3 \pm 0.9$ & $A^{c}$ \\
\hline А883T & $\mathrm{N}$ & 55 & Deleterious 1 & $30.5 \pm 23.3$ & $6 \pm 1$ & $0.8 \pm 0.2$ & $A^{c}$ \\
\hline Y791F & $\mathrm{K}$ & 15 & Deleterious 1 & $30 \pm 18.0$ & $8 \pm 4$ & $0.8 \pm 0.1$ & $A$ \\
\hline V804M & $\mathrm{K}$ & 15 & Deleterious 1 & $18 \pm 4.2$ & $15 \pm 5$ & $1.5 \pm 1.3$ & $A$ \\
\hline M918V & $\mathrm{N}$ & 15 & Deleterious 1 & $16 \pm 8.5$ & $8 \pm 1$ & $1.5 \pm 0.5$ & $A^{c}$ \\
\hline V648I & $\mathrm{N}$ & 0 & Neutral 1 & $12.5 \pm 4.9$ & $10 \pm 4$ & $0.8 \pm 0.2$ & $A^{c}$ \\
\hline T338I & $\mathrm{N}$ & 0 & Neutral 1 & $9.5 \pm 4.9$ & $4 \pm 6$ & $0.9 \pm 0.1$ & $A^{c}$ \\
\hline G691S & $\mathrm{K}$ & 0 & Neutral 2 & $7.5 \pm 0.7$ & $7 \pm 3$ & $1 \pm 0.4$ & NA \\
\hline L790F & $\mathrm{K}$ & 0 & Deleterious 2 & $6.5 \pm 6.4$ & $9 \pm 2$ & $1.3 \pm 0.4$ & $A$ \\
\hline \multicolumn{8}{|l|}{ NIH 3T3 controls } \\
\hline Untransfected & & & & $12.5 \pm 4.9$ & $6 \pm 2$ & $1.7 \pm 0.3$ & \\
\hline $\mathrm{PRC/CMV}$ & & & & $9.5 \pm 3.5$ & $6 \pm 2$ & $1 \pm 0.2$ & \\
\hline Wild-type $R E T$ & & & & $12.5 \pm 0.7$ & $7 \pm 1$ & $1 \pm 0.2$ & \\
\hline
\end{tabular}

FFU, focus-forming unit; ATA, American Thyroid Association; A, low risk; B, intermediate risk; C, high risk; D, highest risk; NA, not applicable because this is a polymorphism and not a transforming mutation.

aTavtigian et al. (2008).

${ }^{b}$ Mathe et al. (2006).

${ }^{\mathrm{c}}$ The level of risk that the new mutations have, defined according to the pathological report and clinical outcome of our patients. 


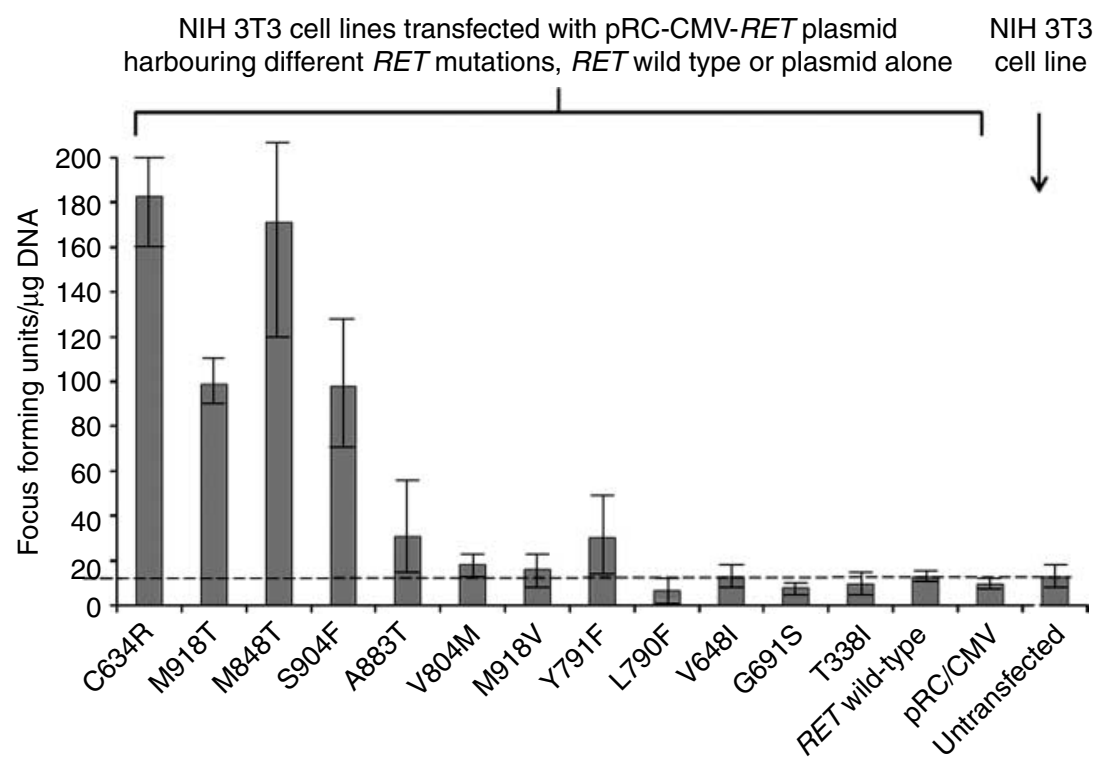

Figure 2 Transforming activities of the six new and the six known missense RET mutations expressed as the number of focus-forming units per microgram of DNA (FFU/ $\mu \mathrm{g}$ DNA) transfected in NIH 3T3 fibroblasts. The dotted line indicates the number of FFU/ $\mu \mathrm{g}$ DNA spontaneously developed in mock- and wild-type transfected and untransfected NIH 3T3 control cell lines $(9.5-12.5 \mathrm{FFU} / \mu \mathrm{g}$ DNA): the $\mathrm{FFU} / \mu \mathrm{g}$ DNA values below this line must be considered as background and not due to a real transforming activity.

the numbers observed in the NIH $3 \mathrm{~T} 3$ cell lines used as controls (6-7 colonies per plate) (Fig. 4 Panel C). The only exception was observed in NIH $3 \mathrm{~T} 3$ fibroblasts transfected with V804M, which produced 10-15 colonies per plate (Fig. 4 Panel B).

\section{Comparison of in silico, in vitro and ATA risk level of known and new germline mutations}

We compared the results of the in silico analysis with the results from the in vitro experiments and the clinical features of our 12 germline mutations in accordance with the level of risk classification of the ATA guidelines (Kloos et al. 2009). For the new mutations, the level of risk has been calculated according to the pathological report and clinical outcome of our patients.

As shown in Table 3, we observed that mutations, both known (C634R and M918T) and new (M848T and S904F), with the highest in silico scores also showed the greater numbers of foci. Conversely, those mutations, both known (G691S and L790F) and new (V648I and T338I), with a 0 score from in silico analysis showed a number of foci similar to controls and can be considered non-transforming. The group of mutations with intermediate in silico scores, ranging from 55 to 15, also showed intermediate numbers of foci. These results suggested a positive correlation between the in silico score and the in vitro transforming activity of the mutations that was confirmed by statistical analysis $(P=0.0005$, by Kendall Rank Correlation test $)$.

With the exception of the two most aggressive known mutations (C634R and M918T), no correlation was observed between the in silico score and the number of colonies obtained in soft agar (Table 3). Conversely, the number of colonies in soft agar correlated with the highest ATA risk levels C and D for C634R and M918T, respectively; all of the others mutations showed a relatively low number of colonies, sometimes lower than the number obtained in the controls, correlating with the lowest ATA risk level A. A similar correlation was observed when correlating the growth proliferation rates in terms of doubling time and the ATA risk levels (Table 3).

\section{Discussion}

The number of newly identified germline mutations has been increasing over the course of time; almost all of them have been detected in apparently sporadic MTC only (Eng et al. 1995b, Berndt et al. 1998, Elisei et al. 2004). Similarly, in our study, the six new mutations have been found in patients without a familial history of MTC and without any other disease typically associated with MEN2 syndromes. These new mutations are usually rare, present only in a few families and within a few family members, raising doubts as to whether they represent the driving force of 


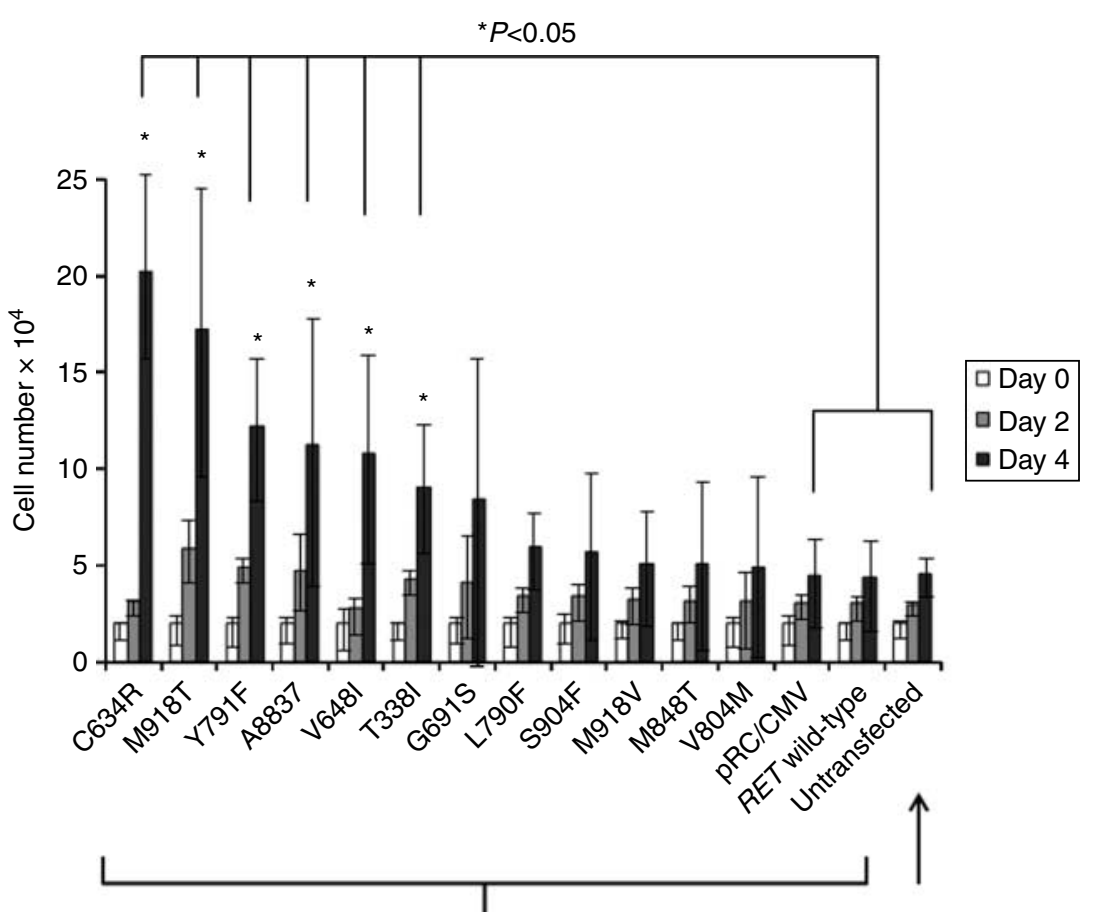

NIH 3 T3 cell lines transfected with pRC-CMV-RET plasmid $\quad$ NIH 3 T3 harbouring different $R E T$ mutations, $R E T$ wild type or plasmid alone cell line

Figure 3 Growth curves of cell lines transfected with the six new and the six known missense RET mutations and controls. All growth curves have been done in triplicate. Only C634R and M918T showed a significantly higher growth rate with respect to control cells. ${ }^{\star} P<0.05$.

the tumoural disease or result from the genetic screening associated with MTC (Orgiana et al. 2004). Indeed, our six new mutations are rare, and four of the six cases are associated with MTC in only one member of the family. Similarly, Frank-Raue et al. (2010) recently described three new germline mutations; each one was detected in one single family and associated with single cases of MTC. Notably, the A883T mutation that we previously reported (Elisei et al. 2004) is associated with MTC only in homozygous subjects, suggesting a low transforming activity of this mutation, which is likely counterbalanced by the normal allele in heterozygous subjects. Other RET mutations, such as Y791F, may also occur more frequently than currently recognised if the development of MTC only occurs in homozygous subjects (Erlic et al. 2010). However, although the Y791F mutation seems to have a high prevalence in the normal German population, none of the six new mutations have been detected in the 200 normal subjects examined in this study; thus, this finding excludes the polymorphic nature of our new mutations and makes the possibility of a homozygous condition in children of non-consanguineous parents very unlikely. Consequently, these new mutations should be considered at least 'potentially' transforming rare allelic variants with activity that might be potentiated by either the homozygous status or compound heterozygosity.

The classical method used to analyse the transforming activity of new mutations is the in vitro FFU assay. In our hands, this assay showed that both the two known highly transforming mutations, C634R and M918T and the two new mutations, M848T and S904F, induced a large number of foci. In the same assay, mutations known to lack any transforming activity, such as G691S (Fugazzola et al. 2008), did not induce FFU. Similarly, two of our new mutations, V648I and T338I, did not show any foci in the in vitro assay. Notably, the V648I mutation was associated with C-cell hyperplasia, not with MTC; the T338I mutation was associated with a case of micro MTC in an adult woman who was identified by serum CT measurement but was clinically silent. Importantly, the T338I mutation has been previously described in Hirschsprung disease (Fitze et al. 2002), which does not belongs to the clinical history of either our patient or her family members, including those harbouring the same mutation.

Among the known mutations, L790F did not induce FFU and had an in silico score of 0 . According to our results, this mutation has no transforming activity, but 

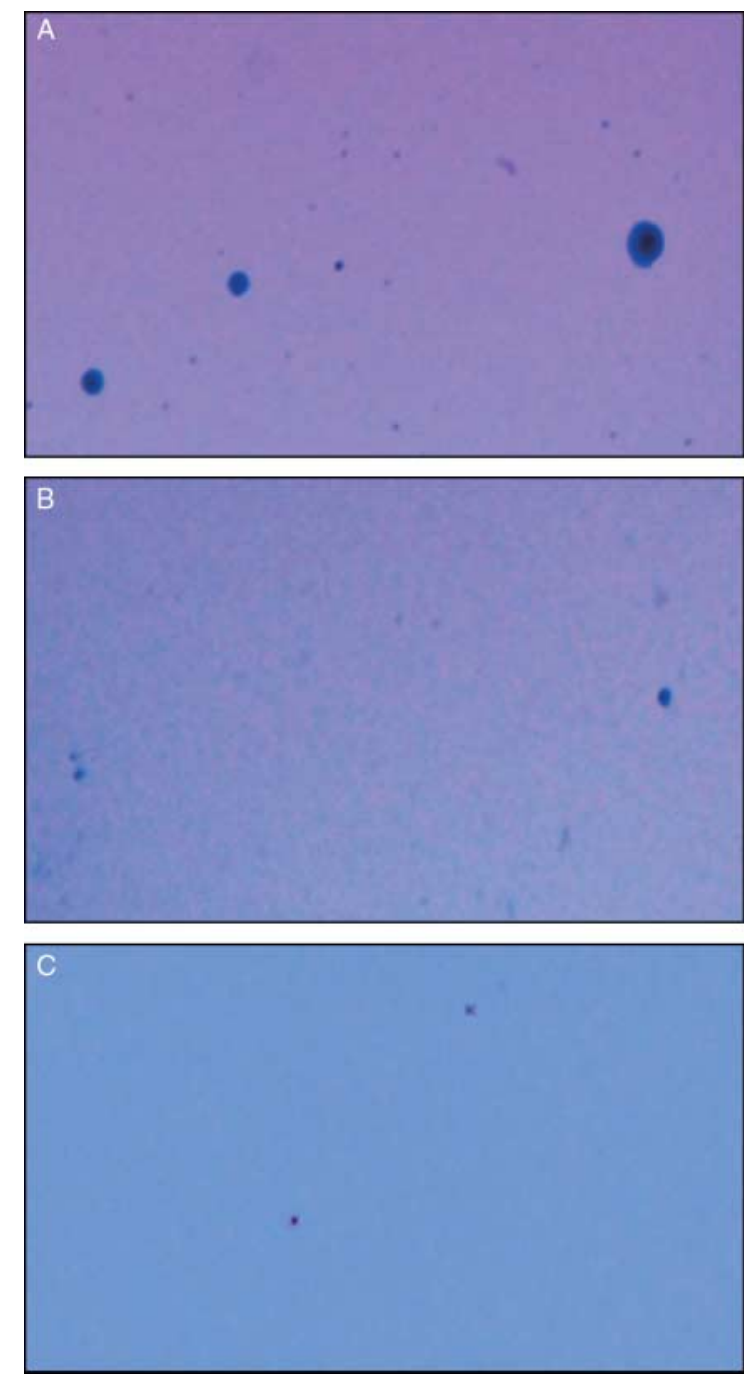

Figure 4 Anchorage-independent growth in soft agar of the $\mathrm{NIH}$ 3T3 cell line transfected with known and new mutations and controls. All experiments have been performed in triplicate.

(Panel A) The C634R mutation produced the largest number of colonies ( $24 \pm 5$ colonies per plate). (Panel B) The V804M

mutation produced an intermediate number of colonies $(15 \pm 5$ colonies per plate), and the majority of the others produced a low number of colonies that were only slightly more numerous than the control. (Panel C) The NIH 3T3 untransfected cells with very few colonies (6-7 colonies per plate).

several German families have been reported with this mutation and FMTC (Gimm et al. 2002). Moreover, in our institutional series, we similarly detected five families out of 103 (4.85\%) with these mutations that were all affected with FMTC (Romei et al. 2011). All but one of our patients carrying this mutation and affected by MTC were cured with surgical treatment, confirming that this mutation belongs to the A category of the ATA risk levels (Kloos et al. 2009). A possible explanation for this apparent inconsistency between the in vitro and the in silico results and clinical findings is that the genetic background of the subject and the entire kindred can play a role in favouring or blocking the transforming activity of the mutation (Lesueur et al. 2006).

Another intriguing new mutation is located on codon 918, which is usually involved in MEN 2B but with a different amino acid change (M918V) from the classical one (M918T). The germline M918V mutation was found by screening an apparently low-risk sporadic case of MTC. The familial screening uncovered the presence of the same mutation in $1 / 3$ of the family members who did not develop the MTC yet, neither at the clinical nor biochemical levels, despite an advanced age. This mutation showed an in silico score of 15 , which was consistent with the results of the focusforming assay (between 15 and $30 \mathrm{FFU} / \mu \mathrm{g}$ DNA). Cirafici et al. (1997) extensively studied the codon 918 and demonstrated that among all the possible substitutions for M918, only the mutation M918T showed a highly transforming activity. According to these findings, we propose that M918V will function similarly to the other low or non-transforming mutations (i.e. T338I and V648I), and only the follow-up of family members with the same mutation will provide further insights into the relationship between these rare variants and the development of MTC.

Concerning the methodology used to demonstrate the transforming activity of the new mutations, we observed that the ability to induce FFU strongly correlated with the in silico score for both the highly transforming and the low or non-transforming mutations. However, this correlation was less evident when considering the functional activity of our RET mutants according to the Mathe et al. (2006) functional prediction approach, but it is worth to note that this last method gave results less defined than the electronic score. Despite these small differences in the in silico assay interpretation, to our knowledge, this study is the first to demonstrate a statistically significant correlation $(P=0.0005)$ between the in silico electronic score and the in vitro FFU assay, thus suggesting the possibility of using the rapid in silico score analysis to predict the transforming potential of newly identified mutations and avoiding the in vitro assay, which is much more expensive and time consuming.

Soft agar assay and growth rate evaluation did not provide much information about the features of the new mutations. The results of both assays appeared to correlate mainly with the most aggressive mutations C634R and M918T, which produced a large number of soft agar colonies and showed the lowest doubling time in culture. All of the other known and unknown mutations showed an insignificant number of soft agar colonies 
and a slow doubling time. Because the results from the soft agar assay and the growth rate evaluation are correlated, these assays appear to be more informative about the aggressiveness of the disease than about the transforming ability of the mutation. In fact, anchorageindependent growth on soft agar is not only one of the most stringent assays for detecting malignant transformation of cells, but there is evidence that the pattern of growth in soft agar can predict biological behaviour and metastatic potential in vivo (Li et al. 1989). According to this interpretation, both C634R and M918T, which are the RET mutations with the highest level of ATA risk, showed also a large number of soft agar colonies and the lowest doubling time.

In conclusion, of the six new mutations we discovered, two of them, M848T and S904F, possess a relatively high transforming activity but a low aggressiveness. The other four mutations, V648I, T338I, A883T and M918V, are low or non-transforming mutations, and their ability to induce tumoural transformation might be related to particular conditions, such as homozygous or compound heterozygous status, or to a predisposed genetic background. Moreover, this study showed that the transforming activity and the aggressiveness of RET mutations can be predicted by the in silico analysis even if in some circumstances, especially in the case of low transforming mutations, the in silico results did not completely correlate with the in vitro assay results. Finally, this new genetic knowledge strongly suggests that subjects harbouring such rare mutations must be followed and treated in tertiary centres by multidisciplinary teams that include endocrinologists, clinical cancer geneticists, endocrine surgeons and psychologists. These individuals should be well trained to handle these patients and their relatives, to avoid unnecessary thyroidectomy while also attending to their psychological status, which is certainly affected by the undefined risk of developing cancer.

\section{Declaration of interest}

The authors declare that there is no conflict of interest that could be perceived as prejudicing the impartiality of the research reported.

\section{Funding}

This study has been supported in part by grants from the Ministero della Istruzione Universitaria e Ricerca Scientifica (MIUR), the Associazione Italiana per la Ricerca sul Cancro (AIRC), the Istituto Toscano Tumori (ITT) and the Ministero della Salute, Progetto Ricerca Oncologica RF-CAM 2006353005. The Department of Endocrinology and Metabolism, University Hospital of Pisa, is a WHO Collaborating Center for the Diagnosis and Treatment of Thyroid Cancer and Other Thyroid Diseases.

\section{References}

Berndt I, Reuter M, Saller B, Frank-Raue K, Groth P, Grussendorf M, Raue F, Ritter MM \& Hoppner W 1998 A new hot spot for mutations in the ret protooncogene causing familial medullary thyroid carcinoma and multiple endocrine neoplasia type 2A. Journal of Clinical Endocrinology and Metabolism 83 770-774. (doi:10. 1210/jc.83.3.770)

Cirafici AM, Salvatore G, De Vita G, Carlomagno F, Dathan NA, Visconti R, Melillo RM, Fusco A \& Santoro M 1997 Only the substitution of methionine 918 with a threonine and not with other residues activates RET transforming potential. Endocrinology 138 1450-1455. (doi:10.1210/ en.138.4.1450)

Donis-Keller H, Dou S, Chi D, Carlson KM, Toshima K, Lairmore TC, Howe JR, Moley JF, Goodfellow P \& Wells SA Jr 1993 Mutations in the RET proto-oncogene are associated with MEN 2A and FMTC. Human Molecular Genetics 2 851-856. (doi:10.1093/hmg/2.7.851)

Elisei R, Cosci B, Romei C, Agate L, Piampiani P, Miccoli P, Berti P, Basolo F, Ugolini C, Ciampi R et al. 2004 Identification of a novel point mutation in the RET gene (Ala883Thr), which is associated with medullary thyroid carcinoma phenotype only in homozygous condition. Journal of Clinical Endocrinology and Metabolism 89 5823-5827. (doi:10.1210/jc.2004-0312)

Elisei R, Romei C, Cosci B, Agate L, Bottici V, Molinaro E, Sculli M, Miccoli P, Basolo F, Grasso L et al. 2007 RET genetic screening in patients with medullary thyroid cancer and their relatives: experience with 807 individuals at one center. Journal of Clinical Endocrinology and Metabolism 92 4725-4729. (doi:10.1210/jc.2007-1005)

Eng C, Smith DP, Mulligan LM, Nagai MA, Healey CS, Ponder MA, Gardner E, Scheumann GF, Jackson CE, Tunnacliffe A et al. 1994 Point mutation within the tyrosine kinase domain of the RET proto-oncogene in multiple endocrine neoplasia type $2 \mathrm{~B}$ and related sporadic tumours. Human Molecular Genetics 3 237-241. (doi:10. 1093/hmg/3.2.237)

Eng C, Mulligan LM, Smith DP, Healey CS, Frilling A, Raue F, Neumann HP, Ponder MA \& Ponder BA 1995 a Low frequency of germline mutations in the RET protooncogene in patients with apparently sporadic medullary thyroid carcinoma. Clinical Endocrinology 43 123-127. (doi:10.1111/j.1365-2265.1995.tb01903.x)

Eng C, Smith DP, Mulligan LM, Healey CS, Zvelebil MJ, Stonehouse TJ, Ponder MA, Jackson CE, Waterfield MD \& Ponder BA $1995 b$ A novel point mutation in the tyrosine kinase domain of the RET proto-oncogene in sporadic medullary thyroid carcinoma and in a family with FMTC. Oncogene 10 509-513.

Erdogan MF, Gursoy A, Ozgen G, Cakir M, Bayram F, Ersoy R, Algun E, Cetinarslan B, Comlekci A, Kadioglu P et al. 2005 Ret proto-oncogene mutations in apparently sporadic Turkish medullary thyroid carcinoma patients: Turkmen study. Journal of Endocrinological Investigation 28 806-809. 
Erlic Z, Hoffmann MM, Sullivan M, Franke G, Peczkowska M, Harsch I, Schott M, Gabbert HE, Valimaki M, Preuss SF et al. 2010 Pathogenicity of DNA variants and double mutations in multiple endocrine neoplasia type 2 and von Hippel-Lindau syndrome. Journal of Clinical Endocrinology and Metabolism 95 308-313. (doi:10. 1210/jc.2009-1728)

Fitze G, Cramer J, Ziegler A, Schierz M, Schreiber M, Kuhlisch E, Roesner D \& Schackert HK 2002 Association between c135G/A genotype and RET proto-oncogene germline mutations and phenotype of Hirschsprung's disease. Lancet 359 1200-1205. (doi:10.1016/S01406736(02)08218-1)

Frank-Raue K, Dohring J, Scheumann G, Rondot S, Lorenz A, Schulze E, Dralle H, Raue F \& Leidig-Bruckner G 2010 New mutations in the RET protooncogene-L881V associated with medullary thyroid carcinoma and -R770Q - in a patient with mixed medullar/follicular thyroid tumour. Experimental and Clinical Endocrinology \& Diabetes 118 550-553. (doi:10.1055/s-0029-1241851)

Fugazzola L, Muzza M, Mian C, Cordella D, Barollo S, Alberti L, Cirello V, Dazzi D, Girelli ME, Opocher G et al. 2008 RET genotypes in sporadic medullary thyroid cancer: studies in a large Italian series. Clinical Endocrinology 69 418-425. (doi:10.1111/j.1365-2265. 2008.03218.x)

Gimm O, Niederle BE, Weber T, Bockhorn M, Ukkat J, Brauckhoff M, Thanh PN, Frilling A, Klar E, Niederle B et al. 2002 RET proto-oncogene mutations affecting codon 790/791: a mild form of multiple endocrine neoplasia type 2A syndrome? Surgery 132 952-959 (discussion 959). (doi:10.1067/msy.2002.128559)

Hecker KH \& Roux KH 1996 High and low annealing temperatures increase both specificity and yield in touchdown and stepdown PCR. BioTechniques 20 478-485.

Kloos RT, Eng C, Evans DB, Francis GL, Gagel RF, Gharib H, Moley JF, Pacini F, Ringel MD, Schlumberger M et al. 2009 Medullary thyroid cancer: management guidelines of the American Thyroid Association. Thyroid 19 565-612. (doi:10.1089/thy.2008.0403)

Lesueur F, Cebrian A, Robledo M, Niccoli-Sire P, Svensson KA, Pinson S, Leyland J, Whittaker J, Pharoah PD \& Ponder BA 2006 Polymorphisms in RET and its coreceptors and ligands as genetic modifiers of multiple endocrine neoplasia type 2A. Cancer Research 66 1177-1180. (doi:10.1158/0008-5472.CAN-05-2995)

Li L, Price JE, Fan D, Zhang RD, Bucana CD \& Fidler IJ 1989 Correlation of growth capacity of human tumor cells in hard agarose with their in vivo proliferative capacity at specific metastatic sites. Journal of the National Cancer Institute 81 1406-1412. (doi:10.1093/jnci/81.18.1406)

Machens A, Lorenz K \& Dralle H 2009 Constitutive RET tyrosine kinase activation in hereditary medullary thyroid cancer: clinical opportunities. Journal of Internal Medicine 266 114-125. (doi:10.1111/j.1365-2796.2009.02113.x)

Mathe E, Olivier M, Kato S, Ishioka C, Hainaut P \& Tavtigian SV 2006 Computational approaches for predicting the biological effect of p53 missense mutations: a comparison of three sequence analysis based methods. Nucleic Acids Research 34 1317-1325. (doi:10. 1093/nar/gkj518)

Mise N, Drosten M, Racek T, Tannapfel A \& Putzer BM 2006 Evaluation of potential mechanisms underlying genotype-phenotype correlations in multiple endocrine neoplasia type 2. Oncogene 25 6637-6647. (doi:10.1038/ sj.onc.1209669)

Mulligan LM, Kwok JB, Healey CS, Elsdon MJ, Eng C, Gardner E, Love DR, Mole SE, Moore JK, Papi L et al. 1993 Germ-line mutations of the RET proto-oncogene in multiple endocrine neoplasia type 2A. Nature $\mathbf{3 6 3}$ 458-460. (doi:10.1038/363458a0)

Nunes AB, Ezabella MC, Pereira AC, Krieger JE \& Toledo SP 2002 A novel Val648Ile substitution in RET protooncogene observed in a Cys634Arg multiple endocrine neoplasia type $2 \mathrm{~A}$ kindred presenting with an adrenocorticotropin-producing pheochromocytoma. Journal of Clinical Endocrinology and Metabolism 87 5658-5661. (doi:10.1210/jc.2002-020345)

Orgiana G, Pinna G, Camedda A, De Falco V, Santoro M, Melillo RM, Elisei R, Romei C, Lai S, Carcassi C et al. 2004 A new germline RET mutation apparently devoid of transforming activity serendipitously discovered in a patient with atrophic autoimmune thyroiditis and primary ovarian failure. Journal of Clinical Endocrinology and Metabolism 89 4810-4816. (doi:10.1210/ jc.2004-0365)

Pasini B, Hofstra RM, Yin L, Bocciardi R, Santamaria G, Grootscholten PM, Ceccherini I, Patrone G, Priolo M, Buys CH et al. 1995 The physical map of the human RET proto-oncogene. Oncogene 11 1737-1743.

Romei C, Cosci B, Renzini G, Bottici V, Molinaro E, Agate L, Passannanti P, Viola D, Biagini A, Basolo F et al. 2011 RET genetic screening of sporadic medullary thyroid cancer (MTC) allows the preclinical diagnosis of unsuspected gene carriers and the identification of a relevant percentage of hidden familial MTC (FMTC). Clinical Endocrinology 74 241-247. (doi:10.1111/j. 1365-2265.2010.03900.x)

Takahashi M, Buma Y, Iwamoto T, Inaguma Y, Ikeda H \& Hiai H 1988 Cloning and expression of the ret protooncogene encoding a tyrosine kinase with two potential transmembrane domains. Oncogene 3 571-578.

Tavtigian SV, Byrnes GB, Goldgar DE \& Thomas A 2008 Classification of rare missense substitutions, using risk surfaces, with genetic- and molecular-epidemiology applications. Human Mutation 29 1342-1354. (doi:10. 1002/humu.20896)

Received in final form 20 June 2011

Accepted 2 August 2011

Made available online as an Accepted Preprint 2 August 2011 